\title{
Dynamic Nuclear Polarization for Neutron Protein Crystallography
}

\author{
Josh Pierce* \\ Oak Ridge National Laboratory \\ E-mail: piercejjeornl.gov
}

\begin{abstract}
The sensitivity of Neutron Macromolecular Crystallography to the presence of hydrogen makes it a powerful tool to study protein structure. This technique is currently limited by the relative low neutron flux provided by even the most modern neutron sources. The strong polarization dependence of the neutron scattering cross section of hydrogen will allow Dynamic Nuclear Polarization to dramatically improve the sensitivity of protein structure measurements. This will enable the use of substantially smaller protein crystals, allowing structure measurements which are currently impossible. We present a proof of concept frozen spin target, built at Oak Ridge National Laboratory to polarize single protein crystals on the IMAGINE beamline at the High Flux Isotope Reactor. The results of the first test on the neutron beam will be discussed, as will planned upgrades to the system.
\end{abstract}

XVII International Workshop on Polarized Sources, Targets \& Polarimetry

16-20 October 2017

Kaist, South Korea

${ }^{*}$ Speaker. 


\section{Introduction}

Macromolecular crystallography is a well established method to determine the structure of proteins. Usually, these measurements are made using X-rays. The extremely high flux available at modern X-ray sources allows structures to be determined in minutes, using extremely small samples. Unfortunately, the nature of photon scattering means that the X-rays scatter preferentially from the heavy atoms in a protein. Current X-ray techniques are less able to locate the lighter atoms in a sample, especially hydrogen, which plays a major role in the function of many proteins. Neutron protein crystallography solves this problem. The neutron scattering cross section for hydrogen is relatively high, giving the neutron a unique sensitivity to the locations of hydrogen in the protein. The wide scale use of this technique is currently limited by the comparatively low flux at neutron scattering facilities. This low flux requires that experimenters take data for much longer periods of time (often weeks) and grow protein crystals one hundred times the size of those used by the X-ray crystallography community. Growing crystals of the required size is a significant technological challenge, one that greatly limits the scientific reach of the technique.

The large spin dependence of the neutron scattering cross section for hydrogen gives a method to solve this problem. In the case of a beam of polarized neutrons, changing the nuclear polarization of the hydrogen atoms can increase the signal size or reduce the backgrounds. There are several well developed techniques for producing highly polarized neutron beams, so all that remains is to implement a system to create high nuclear polarization in samples. Dynamic Nuclear Polarization (DNP) is an obvious choice, producing high hydrogen polarization in a matter of hours. A frozen spin system is needed do the the large acceptance for scattered neutrons required for Neutron Macromolecular Crystallography (NMC).

The potential of DNP enhanced neutron scattering for hydrogenous samples is well known. Small angle scattering experiments have been done using DNP [1][2], and even some tests of inorganic crystals [3], but protein crystallography has not been attempted. A proof of concept system was constructed, and tested on the IMAGINE [4] beamline of the High Flux Isotope Reactor (HFIR) at Oak Ridge National Laboratory (ORNL) in September of 2017.

\section{Spin Dependent Neutron Scattering}

The scattering cross section of a thermal neutron from identical nuclei in a crystal of $N$ unit cells can be written as in equation 2.1 [5].

$$
\frac{d \sigma}{d \Omega}=N\left(\frac{d \sigma}{d \Omega}\right)_{i n c}+N\left(\frac{d \sigma}{d \Omega}\right)_{c o h} \sum_{i} \exp \left(\mathbf{Q} \cdot \mathbf{r}_{\mathbf{i}}\right)
$$

The coherent (2.2) and incoherent (2.3) cross sections can be written in terms of the neutron polarization $p$, the hydrogen polarization $P$, the spin of the nucleus $I$, the spin-independent part of the scattering amplitude $b_{0}$, and the spin dependent part of the scattering amplitude $b$. The terms $b_{0}$ and $b$ have been experimentally determined for large number of nuclei.

$$
\left(\frac{d \sigma}{d \Omega}\right)_{c o h}=b_{0}^{2}+2 b_{0} b I p P+b^{2} I^{2} P^{2}
$$




$$
\left(\frac{d \sigma}{d \Omega}\right)_{i n c}=\frac{b^{2}}{4}\left[I(I+1)-p P I-P^{2} I^{2}\right]
$$

In the limiting case of $p=1$, we can plot the coherent and incoherent cross sections for hydrogen, as in Figure 1. At well optimized beamline, the incoherent scattering from hydrogen is often the largest source of background. Many experiments use deuterated proteins or solvents in an attempt to reduce this background, but in many cases deuteration may be undesirable or impossible. All experiments will benefit from a increase in the coherent cross section, as it will increase the intensity of the measured diffraction. Depending on the relative orientation (aligned or anti-aligned) the cross section for hydrogen can be increased by as much as a factor of twenty, allowing much smaller samples to be used. The best improvement in signal to noise ration may be with aligned or anti-aligned polarization, depending on whether the hydrogen incoherent scattering is the dominant background term, as the incoherent scattering does increase in the anti-aligned case.

\section{Frozen Spin Apparatus}

A prototype frozen spin system for the purpose of polarizing protein crystals has been constructed and test at ORNL. The small size of the samples (typically less that $1 \mu L$ ) allows the use of lower powered refrigerators and microwave sources. The refrigerator used for this prototype was a commercially purchased LD400 dilution refrigerator $^{1}$ with a custom sample interface designed and built at ORNL. The samples were loaded through a gas filled volume, into a Kel-F tube filled with liquid ${ }^{4} \mathrm{He}$. These spaces were not isolated, so a large heat load was present from the superfluid and helium gas in the the sample space and the loading volume. This heat load generally gives a lower limit on the sample temperature of about $200 \mathrm{mK}$.

The polarizing magnet used was a cryogen-free, warm bore, vertical $5 T$ solenoid magnet ${ }^{2}$. The high temperature of the sample prevented the system from entering a true "frozen spin" state. This made it impossible to use a small holding magnet, since the time to switch from the polarizing to holding magnet (limited by the ramp

\footnotetext{
${ }^{1}$ BlueFors Cryogenics Oy

${ }^{2}$ Cryomagnetics, Inc.
}

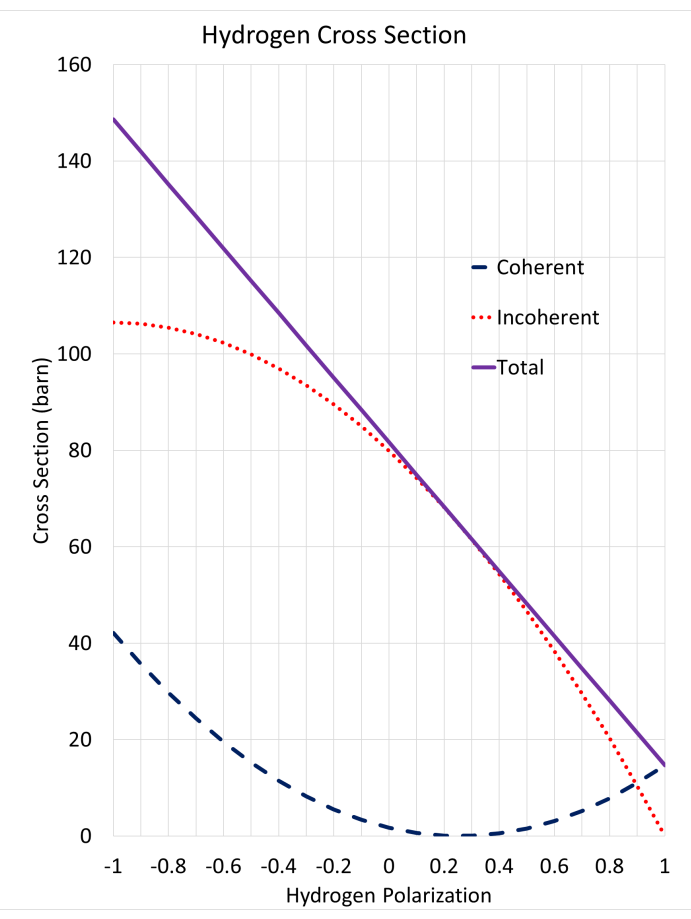

Figure 1: The integrated coherent and incoherent cross sections for polarized neutron scattering off of a polarized hydrogen nucleus, as a function of hydrogen polarization. 
speed of the polarizing magnet) would have been sufficient to lose the majority of the polarization. To overcome this, data was instead taken in the fringe field of the polarizing magnet. The magnet was simply lowered to allow the neutron beam to pass over the top (see diagram in Figure 2).

The microwaves were provided by a solid state $140 \mathrm{GHz}$ microwave source ${ }^{3}$ which was capable of producing up to $300 \mathrm{~mW}$ of microwave power. This was more than sufficient for the very small samples used. The NMR system consisted of a Liverpool [7] q-meter. While the q-meter has sufficient sensitivity to monitor the sample polarization, the small sample size made it impossible to calibrate the NMR system using a thermal equilibrium polarization measurement. Larger (noncrystallized) samples with the same chemical composition as the protein crystals were polarized offline in order to estimate the maximum polarization that could be achieved. These tests showed maximum polarizations of about $50 \%$.

\section{Detector}

The large acceptance detector typically used as part of the IMAGINE beamline does not have sufficient access to allow the system to be used without significant infrastructure upgrades to the beamline. For these tests, it was determined that a single, stand alone detector element should be used. The detector used was a prototype Anger camera, at a distance of approximately $20 \mathrm{~cm}$ from the sample position, with an active area of about $100 \mathrm{~cm}^{2}$. No background subtraction or "flat field corrections" were done, only the the raw output of the detector was used. This results in images that are visually rather poor, but contain a subset of the diffraction pattern.

\section{Sample Material}

Tests were done using crystals of wild type T4-lysozyme. Some tests were done using a different protein crystal (hen-egg lysozyme), but discussion in this report will focus on the T4 lysozyme results.

Low temperature protein crystallography with neutrons can be challenging. This is due to the requirement that the crystal be frozen very quickly. Slow freezing allows

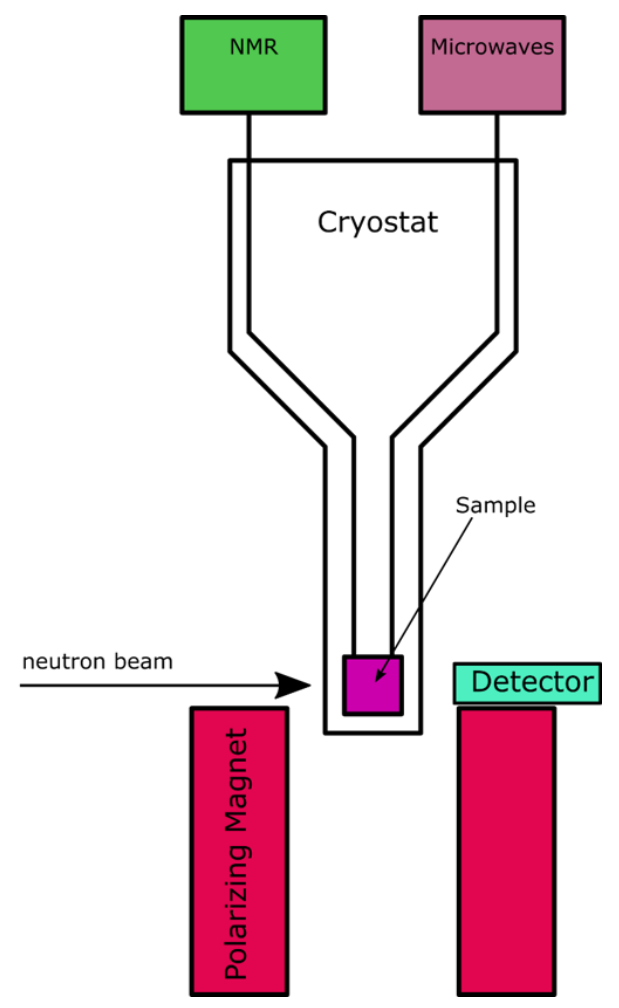

Figure 2: A diagram of the system in data taking mode, showing the magnet lowered to allow the neutron beam to pass over it. After taking data, the magnet would be raised to repolarize the sample.

\footnotetext{
${ }^{3}$ Virginia Diode Incorporated
} 
the formation of water crystals which destroy the order in the protein crystal. Smaller crystals are therefor desirable, since the low volume to surface area ratio makes it easy to freeze them by immersing them in liquid nitrogen. This is at cross purposes with the normal sample requirements for NMC, where larger samples are desirable. In the case of the T4 lysozyme crystals used, studies have been done verifying the viability of low temperature diffraction of crystal of a substantial size [6]. Paramagnetic centers were added to the samples by soaking the crystals in a solution containing $100 \mathrm{mM}$ of hydroxy-TEMPO, with a goal of reaching a final concentration in the crystal similar to the TEMPO concentration used in butanol target polarizing at $5 T[8]$.

\section{First Results}

The first tests at the IMAGINE beamline were remarkably successful. The temperature was quite high $(230 \mathrm{mK})$ which results in a spin relaxation time, $T_{1}$, of about $60 \mathrm{~min}$ in the data taking condition. Data was taken both with the neutron and hydrogen spins aligned, and anti-aligned. Data was generally taken for a time equal to $T_{1}$, meaning that the average polarization for any data set is substantially lower than the max polarization. Since the maximum polarization is estimated to be $50 \%$, the best enhancement is expected to be when the neutron and hydrogen spins are anti-aligned (see Figure 1). During these tests, individual parts of the diffraction pattern sowed an increase in intensity of as much as a factor of three. An example comparing aligned and anti aligned diffraction data is shown in Figure 3.
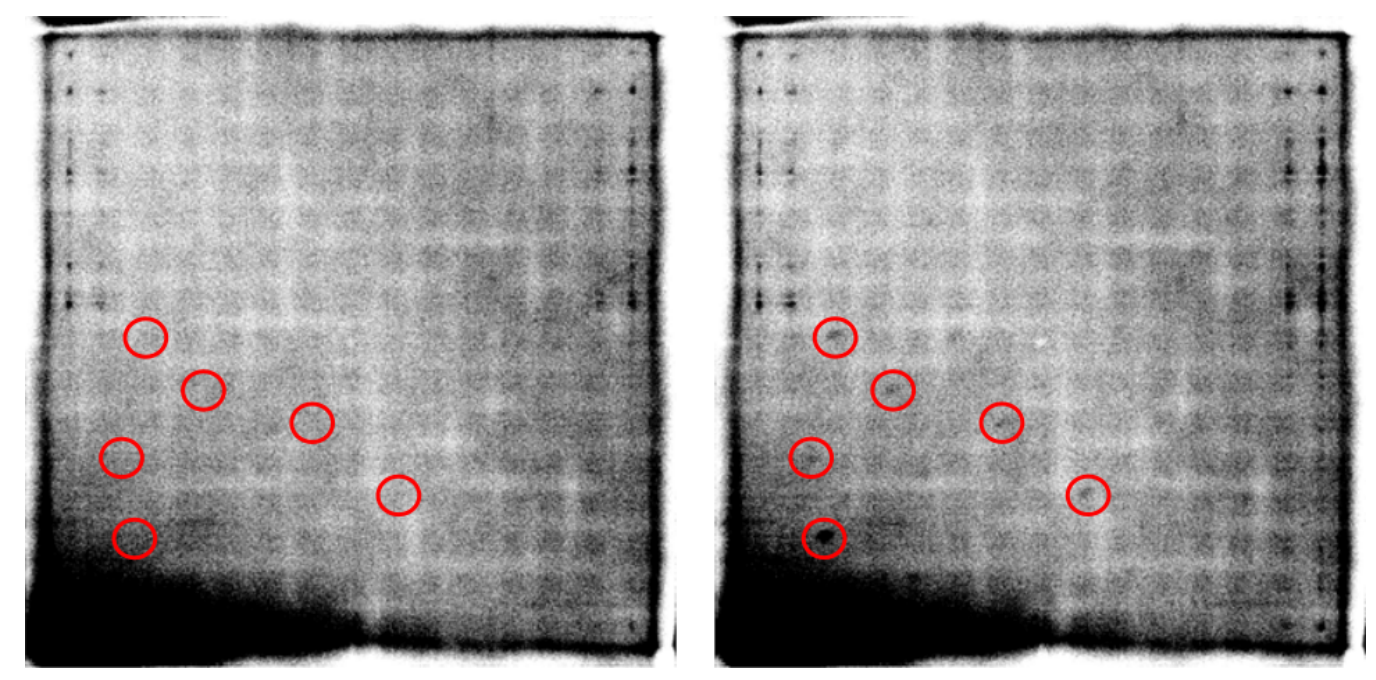

Figure 3: Left, diffraction data taken with the sample and the neutron polarized in the same direction. Right, data taken with the same polarization, but with the spins anti-aligned. Circles show the location of the diffraction data. For the aligned case, the hydrogen cross section has reduced to the point that the diffraction pattern is indiscernible, where as in the anti-aligned case, the diffraction pattern has started to appear.

\section{Outlook}

Another test run was conducted in November of 2017, using the same apparatus, and a larger number of crystals. Analysis of the data is currently underway. Moving forward, the primary 
goal is to reduce the sample temperature in order to have system operate as a true frozen spin system. This will require using a sealed sample space, similar to the one used in the FROST target [8]. These modifications are currently under construction and will be ready for in beam tests in the coming year. Lowering the temperature to $50 \mathrm{mK}$ would effectively double the average polarization, and allow data to be taken for a much longer period of time. Tests with the new system will highlight the potential for DNP enhance NMC, and will inform the design of a potential new protein crystallography beamline, which could incorporate DNP to allow measurements to be made in less time, on crystals orders of magnitude smaller than those current used.

\section{Acknowledgments}

Research sponsored by the Laboratory Directed Research and Development Program of Oak Ridge National Laboratory, managed by UT-Battelle, LLC, for the U. S. Department of Energy. This research was done in collaboration with the members of the DyPol LDRD team. The construction and installation of the IMAGINE beam line was partly supported by NSF grant CHE-0922719.

\section{References}

[1] Zhao, J., and H. B. Stuhrmann. "The in situ structure of the L3 and L4 proteins of the large subunit of E. coli ribosomes as determined by nuclear spin contrast variation." Le Journal de Physique IV 3.C8 (1993): C8-233.

[2] Noda, Y., et al. "Inhomogeneous dynamic nuclear polarization of protons in a lamella-forming diblock copolymer investigated by a small-angle neutron scattering method." Journal of Applied Crystallography 44(3): 503-513. (2011).

[3] Piegsa, F. M., et al. "Polarized neutron Laue diffraction on a crystal containing dynamically polarized proton spins." Journal of Applied Crystallography 46(1): 30-34 (2013).

[4] Meilleur, Flora, et al. "The IMAGINE instrument: first neutron protein structure and new capabilities for neutron macromolecular crystallography." Acta Crystallographica Section D: Biological Crystallography 69.10 (2013): 2157-2160.

[5] Abragam, A. "The principles of nuclear magnetism.” Oxford university press. (1961).

[6] Li, Le, et al. "Neutron crystallographic studies of T4 lysozyme at cryogenic temperature." Protein Science (2017).

[7] G.R. Court, D.W. Gifford, P. Harrison, W.G. Heyes, and M.A. Houlden, Nuclear Instruments and Methods in Physics Research Section A 324 (1993) 433.

[8] C.D. Keith, et al., Nuclear Instruments and Methods in Physics Research Section A 684 (2012) 27. 